\title{
Efecto de los derivados financieros en el valor de las empresas latinoamericanas
}

\author{
Effect of financial derivatives on the value of Latin America companies
}

\author{
Jhon Pinilla Bedoya , Juan David Martínez Muñoz \\ Universidad de Antioquia, Colombia
}

Recibido el 12 de febrero de 2019; aceptado el 26 de julio de 2019

Disponible en Internet el: 30 de julio de 2019

\section{Resumen}

El presente artículo tiene como finalidad conocer si la decisión de usar o no derivado financieros tiene un efecto en el valor de las organizaciones, así como su comportamiento y magnitud dentro del esquema empresarial. Para ello, se dispuso de una muestra de 60 compañías que negocian en ADR's y que pertenecen a los países de Argentina, Brasil, Chile, Colombia, México y Perú. Se usaron 3 modelos basados en la metodología de panel de datos por efectos fijos, en los cuales se tomaron para cada uno de ellos las variables independientes: Q de Tobin, índice RONA y Flujo de Caja, al suponer que estos indicadores miden de una u otra manera el valor una compañía. Los resultados mostraron consistencia al establecer que, para la muestra seleccionada, el uso de los derivados financieros no tiene un impacto significante en el valor de las mismas, y que, por el contrario, a lo pensado, tampoco agregan valor. En cuanto a las estrategias mantenidas en estos portafolios por parte de las compañías objeto de análisis, se encontró que pocas veces estas son importantes dentro de la estructura financiera empresarial, y que a la hora de la verdad sus resultados son cuestionables.

Código JEL: G14, G23, G32

Palabras clave: Derivados financieros; Valor de las empresas; RONA; Q de Tobin; Flujo de caja

* Autor para correspondencia

Correo electrónico jhonp92@hotmail.com (J. Pinilla Bedoya).

La revisión por pares es responsabilidad de la Universidad Nacional Autónoma de México. 


\begin{abstract}
This paperwork has as goal to determine whether the decision to use financial derivatives or not has an effect on the value of organizations, as well as its behavior and magnitude within the business structure. For this, we had a sample of 60 companies that trade in ADRs and that belong to the countries of Argentina, Brazil, Chile, Colombia, Mexico and Peru. Three models were used based on the data panel for fixed effects, in which the independent variables: Tobin Q, RONA index and the Cash Flow, were taken for each of them, assuming that these indicators measure of somehow the value a company. The results showed consistency when establishing that the use of financial derivatives does not have a significant impact on their value, and on the contrary to what was thinking, they do not add value either. Regarding the strategies maintained in these portfolios by the companies, it was found that these are barely important within the corporate financial structure, and their results are questionable.
\end{abstract}

JEL code: G140, G230, G320

Keywords: Financial derivatives; Company values; RONA; Tobin’s Q; Cash flow

\title{
Introducción
}

La teoría financiera moderna en sus primeros desarrollos socavó fuertemente la necesidad de poseer un sistema de gestión de riesgos por parte de las organizaciones. Esta idea, enmarcada en los preceptos de Modigliani y Miller (1958), demeritaba el uso de estrategias tendientes a gestionar los riesgos a los cuales podrían enfrentarse las compañías dentro de un mercado, pues se suponía que estas últimas se encontraban en manos de inversores óptimamente diversificados que operaban en mercados completos, eficientes y sin fricciones, y que por lo tanto, estos llegarían a eliminar cualquier problema a través de sus opciones de cartera (Cummins, Phillips, \& Sthepen, 2001), fijando así, la percepción de que cualquier acción que una organización pudiera realizar a través de la administración de riesgos, podría ser lograda por el inversor con anterioridad (Bartram, Brown, \& Conrad, 2011).

En años más recientes, dichas teorías han ido cediendo, dando paso a un nuevo acervo teórico donde la práctica de la gestión de riesgos se hace cada vez más importante, pues se considera que con su apoyo se puede incrementar el valor de las organizaciones al combatir las imperfecciones de los mercados, tales como los costos de bancarrota o de estrés financiero, los efectos impositivos de los diferentes programas tributarios, los problemas de subinversión, los costos de agencia y las asimetrías de información (Bartram, Brown, \& Fehle, 2009). A esto, autores como Kozikowski (2007), Gay y Nam (1998) y, Berkman y Bradbury (1996), mencionan que siempre que existan dichas imperfecciones, las conjeturas de Modigliani y 
Miller no se cumplirían, y por lo tanto, las medidas contra riesgos son factibles y podrían impactar positivamente en las organizaciones.

Es así, como los agentes han tomado todo tipo de garantías para asegurar el buen desarrollo de sus operaciones con miras a proteger y maximizar el valor empresarial dentro de mercados que distan de ser perfectos (Corzo \& Balbi, 2007), y para ello, han hecho uso de algunos mecanismos que les ayudarán a gestionar sus riesgos, entre los cuales existen los llamados derivados financieros, herramientas que han encontrado su lugar en los últimos años, y que cada vez constituyen una característica más de los mercados mundiales, pues apoyan a las compañías a la hora de navegar en medio de ambientes de incertidumbre y poca predictibilidad, repercutiendo así en una mejor gestión del futuro financiero (Place \& Gray, 2003).

A pesar de que se ha tenido como verdadera la anterior presunción, en la literatura académica se pueden encontrar posiciones contrarias a la mencionada idea, sin embargo, estas no son conclusivas pues debido a los pocos estudios realizados, no se ha posibilitado llegar a un consenso.

Por lo tanto, el presente artículo tiene como objetivo ayudar a la construcción de un acervo teórico más completo sobre el tema de los derivados financieros y su efecto en el valor de las empresas, enfocándose para ello en un mercado latinoamericano, usando como variables dependientes en los modelos la Q de Tobin, el índice RONA y el Flujo de Caja, como indicadores de valor. Del mismo modo, se precisa en el hecho de que se poseen los valores de las posiciones netas en derivados y sus resultados generados para cada año, por lo cual, se da mayor alcance al modelo al no depender solamente de una variable dummy para derivados sino también de las cuantías reportadas por las organizaciones de la muestra en cuanto a este tema.

Por último, el trabajo está organizado en 4 secciones, comenzando por un estado del arte a modo de referente y base de la presente investigación y donde se exponen una serie de estudios del mismo corte. En un segundo y tercer aparte se especifica la metodología usada, el modelo construido, y el análisis de los resultados. Por último, se dan algunas consideraciones finales y recomendaciones para futuras investigaciones.

\section{Sobre algunos estudios en la literatura especializada}

No son realmente amplias las investigaciones realizadas en torno al tema de los derivados financieros y al valor de las empresas, sin embargo, puede encontrarse algunas publicaciones en varias revistas académicas, las cuales reportan diferentes posiciones en cuanto a la relación de estas variables. Algunos autores como Allayannis y Weston (1998), Saito y Schiozer (2009), y Graham y Smith (1999), reafirman el efecto positivo que tienen las estrategias con derivados financieros en el valor de las organizaciones, mientras que otros como Guay 
y Kothari (2002) y Bartram, Conrad y Brown (2011) no encuentran muy significante esta relación. Entre tanto, autores como Jin y Jorion (2006) hallan sobre este mismo tema una relación negativa. Por lo variado de los resultados, parece importante exponer las posiciones y hallazgos de algunos investigadores con el fin de consolidar en un solo lugar las diferentes posturas encontradas en la literatura especializada.

Allayannis y Weston (1998) parten de una muestra de 720 grandes empresas no financieras estadounidenses entre los años 1990 y 1995, y encuentran que aquellas organizaciones que utilizan derivados financieros de divisas tienden a ser más valiosas que aquellas que no los usan. En promedio, observan un incremento en el valor de cerca de $4.87 \%$, lo cual traduce alrededor de 153 millones de dólares. Por su parte, Graham y Rogers (2002) establecen una relación positiva entre el uso de derivados y la capacidad de endeudamiento, lo que resulta en un aumento del valor empresarial del $1.1 \%$, debido a los beneficios impositivos de poseer una deuda financiera mayor y a la reducción de la volatilidad de las ganancias, para ello se basan en una muestra de 3.232 compañías que presentan el informe 10-K ante la Securities and Exchange Commission de Estados Unidos.

Guay y Kothari (2002) enfocan su investigación en torno a 413 corporaciones no financieras que negocian en sus acciones en el mercado estadounidense, evaluando en ellas la sensibilidad del flujo de caja y el valor de la cartera de derivados a cambios extremos en los precios de los activos subyacentes de los portafolios de derivados poseídos. Revelan entonces que, a raíz de esto, se pueden generar impactos positivos de cerca de 15 millones de dólares en flujo de caja, o de 31 millones de dólares en el valor de la cartera de derivados. A pesar de esto, afirman que dichas cantidades son pequeñas para estas empresas, y son totalmente modestas en comparación con todas las exposiciones a riesgo que se tienen, cubriendo en todo caso solo del $3 \%$ al $6 \%$ de estas. Concluyen su estudio diciendo que los derivados financieros son simplemente un ajuste pequeño a los programas de riesgos de las empresas.

Sobre los resultados presentados por Allayannis y Weston (1998), Guay y Kothari (2002) expresan que dichos hallazgos pueden generar dudas, debido a que se sugiere que los aumentos en el valor de las empresas documentadas pueden estar impulsados por otras actividades de administración de riesgos, tales como coberturas operativas, las cuales están correlacionadas positivamente con el uso de derivados financieros. En consonancia con Guay y Kothari (2002), Brown (2001) llega a similares conclusiones al evaluar la gran multinacional HDG Inc., pues halla que el uso de derivados financieros posee en esta un limitado efecto en el flujo de caja, y que las teorías tradicionales de gestión de riesgo difícilmente explican por qué una compañía se ve motivada a usar estos instrumentos ante el poco impacto que producen.

Saito y Schiozer (2009) examinan una muestra de 57 empresas pertenecientes a los países de Argentina, Brasil, Chile y México para el año 2004. En su estudio, concluyen que el uso de derivados financieros de cobertura para el riesgo cambiario es económicamente significativo, 
y pueden incrementar el valor de una compañía entre 3\% y 7.6\%. Esto debido en su mayor parte a que dichas herramientas pueden generar flujos de caja equivalentes al $83.7 \%$ de los gastos financieros, $61.1 \%$ de gastos de capital (en el caso de cambios extremos en el valor de los activos subyacentes) y $29.4 \%$ de las ganancias antes de impuestos e intereses. Más recientemente, los mismos autores renuevan su investigación tomando ahora una muestra de 55 compañías para los años 2001 a 2004 (Schiozer \& Saito, 2014), y aun así llegan a iguales conclusiones, solo que para esta ocasión los porcentajes varían significativamente, siendo estos de $30 \%$ a $50 \%$ de los gastos financieros, más del 100\% de los gastos de capital, y del $8 \%$ al $40 \%$ de las ganancias antes de impuestos e intereses. Hay que aclarar que la metodología de panel de datos se mantuvo de un estudio a otro.

Estos últimos autores también encuentran evidencia de que los derivados financieros impactan positivamente al reducir los costos de estrés financiero, mitigar los problemas de subinversión e información asimétrica, asegurar la generación interna de efectivo e incrementar las posibilidades de crecimiento, validando así las teorías antes mencionadas, exceptuando el caso de la reducción de impuestos por utilización de dichos instrumentos. Con respecto a este tema impositivo, Graham y Smith (1999) demuestran que las empresas con programas tributarios convexos pueden aminorar las cargas imponibles en casi un $5.4 \%$ en promedio, alcanzando valores cercanos al $40 \%$ en casos extremos (bajo el régimen fiscal estadounidense), todo gracias al uso de instrumentos derivados.

En su investigación, Saito y Schiozer (2009) manifiestan que sus resultados difieren sustancialmente de los hallazgos de Guay y Kothari (2002), debido a que: las volatilidades de tasa de interés y tipo de cambio son mucho más grandes en los países objeto de estudio; las empresas de esta latitud buscan una mayor proporción de fondos en el extranjero a falta de fondos internos; y la práctica de cobertura es más común y se tiene mayor información en los años escogidos.

Jin y Jorion (2006) sugieren, al igual que el estudio anterior, que muchas investigaciones solo proveen de evidencia indirecta entre las coberturas y el incremento en el valor de las organizaciones. Expresan, que el uso de coberturas no siempre impacta positivamente, y dan como argumento que si los riesgos son identificables y fácilmente protegidos por parte de los inversionistas, la prima por cobertura tiende a desaparecer gracias a la diversificación, por lo que cualquier estrategia de este tipo irá en contravía a su objetivo, convirtiéndose en innecesarias. A estas conclusiones llegan luego de evaluar 119 empresas pertenecientes a la industria del petróleo y gas estadounidense, encontrando que las empresas que se cubren son un $4 \%$ menos valiosas que las que si lo hacen.

Como contrapeso, Cummins, Phillips y Smith (2001) evalúan una muestra de 1216 empresas aseguradoras de vida y 1668 compañías de seguro de propiedad que reportaron información al NAIC (National Association of Insurance Commissioners) de Estados Unidos en el año 
1994. De su estudio extraen que aquellas aseguradoras que se cubrían eran más valiosas que las que no al no tener que enfrentar costos de dificultades financieras, mejorar la eficiencia de la rentabilidad, y reducir la volatilidad de los activos, la liquidez y el riesgo de tipo de cambio.

Guay (1999) para una muestra de 254 corporaciones no financieras estadounidenses, encuentra que el inicio de un programa de control de riesgos con derivados financieros puede reducir, al año siguiente de su implementación, entre el 5\% de la volatilidad del retorno de la acción, el $22 \%$ de la exposición al riesgo de tasa de interés y en $11 \%$ la exposición al riesgo cambiario para estas compañías, lo cual contribuye a su gestión empresarial positivamente, y por ende, a ser más apreciadas en el mercado.

Estos resultados positivos son potenciados con el estudio de Murillo, Chen, Yue y Hong (2011), los cuales suministran información de 1185 empresas y 2718 contratos de préstamo suscritos al mercado estadounidense, hallando que el uso de derivados financieros de cobertura puede ayudar a aumentar en $13 \%$ la inversión, reducir el costo de la deuda en $29 \%$ y aminorar las restricciones de capital en $20 \%$. Si bien esta investigación no determina una relación directa entre los derivados y el valor empresarial, propone una relación entre su costo y beneficio, planteando así que en promedio las coberturas pueden dar ganancias netas de $4.7 \%$ del ingreso anual.

Bartram, Conrad y Brown (2011), por su parte, apoyan el argumento de que el valor de las compañías no se ve impactado significativamente por el uso de derivados como lo establecen otros estudios, sin embargo, si reduce fuertemente el riesgo sistemático, el riesgo total y la volatilidad de los flujos. Para llegar a estas deducciones, se amparan de una muestra de 6888 empresas de 47 países diferentes.

En el Reino Unido, Ahmed, Azevedo y Guney (2013) consideran una muestra de 288 compañías que cotizan en la London Stock Exchange, y dividen su investigación por clase de derivado (Forward, Futuros, Opciones y Swaps) y tipo de subyacente (Tasa de cambio, tasa de interés y commodities) con relación a medidas de valor tales como el ROIC, el ROA y la $\mathrm{Q}$ de Tobin. De esta manera hallan que: la cobertura de riesgo cambiario para todos los tipos de derivados es positiva y significativa con respecto al rendimiento y valor de la empresa; la cobertura de commodities es positiva pero no significativa, excepto en el caso de los swaps, donde aun siendo positiva si posee significancia; y por último, la cobertura de tasa de interés es negativa y significativa, menos en el caso de los forward, donde la relación es positivamente significativa con respecto a las variables dependientes.

Para 227 organizaciones de Hong Kong y China, Clark, Judgeb y Ngaib (2006) validan el efecto del uso de derivados financieros con relación al valor empresarial. En su estudio estos los autores hallan que las estrategias de cobertura en esta parte del mundo aumentan el valor de las empresas en $0.88 \%$ (Empresas de Hong Kong) y 0.56\% (Empresas chinas), todo gracias al incremento en la capacidad de endeudamiento y la protección ante costos de dificultades financieras. 
En Norteamérica, Tim y Chitru (2006) usan una muestra de 92 compañías de minas de oro, y hallan que el uso de derivados financieros posibilita la generación de significativos flujos de caja tanto en épocas que el mercado está al alza como a la baja, resultando en una valorización de estas. En promedio, estas mineras generan 11 millones de dólares de flujo de caja o bien 24 dólares por onza de oro cubierta, además de ingresos netos por 3.5 millones de dólares. Estas cifras se derivan de las primas de riesgo presentes en el mercado del oro, el grado de cobertura de las compañías, y las expectativas por parte de los administradores en cuanto a esta commoditie, dado que esta última repercute seriamente en las estrategias de riesgo con coberturas (Brown \& Crabb, 2002). Adicionalmente, Tim y Chitru (2006) mencionan para las mineras, el uso de derivados reduce la exposición del precio del oro en un 54\%, lo cual ciertamente va en contravía a investigaciones tales como la de Hentschel y Kothari (2001), quienes encuentran poca o nula diferencia en la exposición de riesgo o volatilidad que enfrentan las empresas que usan o no derivados.

Sarmas (2005) provee de una nueva perspectiva a la investigación de los derivados financieros, evaluando la decisión de cubrirse o no ante ambientes de auge y recesión económica. Para ello, se apoya en una muestra constituida por organizaciones que hacen parte del índice S\&P500 durante los años 1999 (época de bonanza) y 2001 (época de contracción). Se llega a hallazgos que sustentan que las empresas que decidieron cubrirse tanto en años de prosperidad como de adversidad muestran un valor mayor que el de sus contrapartes. Los autores enfatizan que dichos resultados se deben en gran parte por los beneficios impositivos de las coberturas y el alivianar los costos de distress.

Allayannis, Lel y Miller (2012) integran el concepto de gobierno corporativo al análisis de los derivados financieros y el valor empresarial, concluyendo que las empresas que poseen un gobierno corporativo más fuerte y que están en países con una mayor preocupación y proteccionismo por los inversionistas, tienden a usar más derivados, aumentando en todo caso el valor de dichas organizaciones gracias a la reducción de costos de agencia. Se observa entonces, una prima de $10.7 \%$ en el valor de las empresas, una cifra mucho mayor a estudios previos de Allayannis, lo cual se da, según palabras de los mismos autores, porque la muestra de 372 compañías está conformada por agentes económicos de diferentes partes del mundo que poseen una mayor exposición a los riesgos cambiarios. Estos hallazgos son consistentes con Fauver y Naranjo (2010) al examinar una muestra de 1746 firmas que poseen su casa matriz en Estados Unidos, pues los mismos mencionan que la relación entre derivados de cobertura y valor empresarial es negativa cuando estas poseen gobiernos corporativos débiles o con serias deficiencias.

Para una muestra de 312 empresas inmersas en el mercado estadounidense entre los años 1992 y 1996, Deshmukh y Stephen (2005) estudian el impacto de los derivados financieros en el flujo de caja. Esta investigación presenta entre sus resultados una reducción sustancial 
de la volatilidad del flujo de caja y de los costos asociados a la subinversión para aquellas compañías que se cubren con relación a las que no.

Adentrándonos a Latinoamérica, Coronas (2012) evalúa el posible efecto de los derivados financieros (tanto de cobertura como de especulación) en la evolución de las cotizaciones bursátiles de empresas no financieras mexicanas que hacen parte del cálculo del índice de precios y cotizaciones de la Bolsa Mexicana de Valores (BMV). De esta manera, y usando una metodología de datos de panel de efectivos fijos y aleatorios, encuentra que la utilización de derivados financieros posee una influencia positiva y significativa con respecto a los valores de la cotización de las empresas, esto debido a la reducción de la exposición al riesgo cambiario.

Fierros Villanueva (2012) toma en cuenta 69 empresas de la BMV para los años que van desde el 2000 a 2011, y usando también modelos de datos de panel, encuentra que las estrategias de gestión de riesgos con derivados no tienen un impacto positivo y significativo en el valor de las empresas, lo cual va en contravía a los resultados del estudio anterior.

En Chile, Castillo y Moreno (2008) llegan a conclusiones iguales a las de Coronas (2012) y Fierros (2012), al mencionar que las empresas chilenas se ven afectadas negativamente en su valor por el riesgo cambiario al cual se encuentra expuestas, es decir, que con la puesta en práctica de estrategias de cobertura con derivados, se puede mitigar dicho riesgo e impactar positivamente el valor de estas organizaciones. Los autores aclaran también que una cosa es el uso de derivados y otra el monto bruto de los mismos, expresando que dentro del modelo propuesto, esta última variable no fue significativa.

Para este mismo país, Cowan, Hansen y Herrera (2006) indican que el uso de derivados cambiarios es solo un complemento de la gestión de riesgo, pues en las empresas chilenas es la deuda extranjera la más usada como mecanismo de cobertura cambiaria, a pesar de que ha ido en caída en los últimos años. Del mismo modo, concluye que el uso de derivados está relacionado grandemente con la cantidad de activos, deuda y nivel de ingresos en moneda extranjera.

En Brasil, Berrospide, Purnanandam y Rajan (2008) consideran una muestra de 167 compañías para los años comprendidos entre 1997 y 2004, y encuentran que las empresas que usan derivados financieros de cobertura cambiaria son de $6.7 \%$ a $7.8 \%$ más valiosas que aquellas que no se cubren. Argumentan que esta prima se da debido a que dichas organizaciones aumentan su capacidad de deuda, evitan enfrentar costos de bancarrota o distress, cuidan el capital de inversión, y evitan problemas de subinversión,

Por último, en Colombia, Giraldo y Otros (2017) analizan mediante un modelo de datos de panel de efectos fijos y aleatorios, una muestra de 39 compañías no financieras para los años comprendidos entre 2008 a 2014, con la finalidad de observar si el uso de derivados financieros repercute en el valor de las mismas (mediante la Q de Tobin). De esta forma, estiman que las organizaciones que utilizan derivados incrementan su valor poco más del 
$6.4 \%$ con respecto de aquellas que no, además de encontrar una relación positiva entre el apalancamiento total, la capitalización bursátil, la rentabilidad neta y el margen EBITDA, con relación al valor empresarial.

Como pudo observarse, los estudios realizados en torno a los derivados financieros encuentran evidencias de carácter mixto, a esto podríamos traer a colación lo dicho por Dhanani, Fifield, Helliar y Stevenson (2007), que expresan que el efecto de las coberturas en el valor de las organizaciones podría variar según los países, los regímenes fiscales, las imposiciones regulatorias, y demás condiciones; o también lo mencionado por Scordis, Barrese y Wang (2008) que manifiestan que la falta de consenso empírico entre las investigaciones hechas sobre el tema no es de sorprender dado el mosaico de datos que muchos estudios recogen, lo que a la final fuerza y limita los análisis, y hace difícil la comparación y generalización de los resultados entre empresas o inclusive, entre industrias. A consideración de los autores, dicha falta de consenso, adicional a lo expuesto en este párrafo, se ve remarcado al integrar en el análisis temas como la disparidad de la cultura financiera entre épocas y latitudes del mundo, un creciente boom en el uso de derivados que antes no existía, una tecnología que posibilita realizar transacciones al instante y por parte de una base de operadores más grande, contar con mejor información con el pasar del tiempo y, lógicamente, el uso de diferentes supuestos o variables (que incluso al ser las mismas, pueden tener diferentes formas de cálculo). No se remarca el uso de distintas metodologías al ser preponderante los cálculos con panel de datos en la literatura revisada.

Como hipótesis, los autores manifiestan que el usar una buena estrategia de derivados financieros debería de proveer a las empresas de una prima en su valor, ya sea por los beneficios esbozados al inicio de este trabajo, o por el simple hecho de cubrirlas de la exposición ante variables de mercado, o bien por el menor costo que revisten el utilizarlos en comparación a otras estrategias de cobertura (Geczy, Bernadette, \& Schrand, 1997). A pesar de esto, se considera que estos instrumentos no son la panacea, y más bien son una parte integral de todo programa de riesgos, y que dichas herramientas por sí solas no llegarían a cubrir la totalidad de riesgos existentes en una compañía.

\section{Metodología y modelación}

Teniendo como base la investigación de Saito y Schiozer (2009), el presente trabajo tuvo como foco de estudio el mercado Latinoamericano, representado por los países de Argentina, Brasil, Chile, Colombia, México y Perú, por ser estas naciones aproximadamente el $90 \%$ del total del mercado financiero de esta región. Como muestra se tomaron las organizaciones de los países mencionados que negocian ADR's (American Depositary Receipt) en las princi- 
pales bolsas de Estados Unidos (NYSE y Nasdaq), excluyendo para esto, las compañías del sector financiero (Bancos, fondos mutuos de inversión, corporaciones de crédito, entre otros).

Específicamente, la muestra se constituyó de 60 organizaciones divididas en: 11 empresas de Argentina, 23 de Brasil, 8 de Chile, 3 de Colombia, 12 de México y 3 de Perú. Por su parte, y de acuerdo con su sector de procedencia, las mismas se catalogan así: 13 compañías del sector de bienes de consumo y retail, 7 del sector minero, 8 del sector de aerolíneas, 5 del sector constructor, 2 del sector de servicios públicos, 4 del sector de los hidrocarburos y gas, 10 del sector de energía, 2 del sector químico, 2 del sector agricultor, y 7 del sector de las telecomunicaciones.

En cuanto a la información financiera y bursátil de estas compañías, esta se extrajo en su mayoría de los informes 20-F y 10-K, presentados ante la Securities and Exchange Commission (SEC) de los Estados Unidos para los años comprendidos entre el 2013 y 2017. Igualmente, se consideraron informes de carácter financiero, de gestión, de responsabilidad social y de resultados, así como presentaciones y audios en convenciones internacionales, generalmente exhibidos en las páginas Web para inversores de estas empresas. Por último, se recurrió al software Economatica con la finalidad de completar información faltante o corroborar algunos datos importantes tales como la capitalización bursátil y ciertos índices de rendimiento.

Respecto al componente metodológico, esta investigación supuso el uso de modelos econométricos bajo el método de análisis de panel de datos toda vez que sus características se ajustan más a la forma en como está construida la muestra (observaciones de corte transversal a lo largo del tiempo) y los beneficios que este supone, entre ellos: proporcionar una mayor cantidad de datos informativos, más variabilidad, menos colinealidad entre variables, más grados de libertad y tener en cuenta de manera explícita la heterogeneidad de las unidades. En conclusión, una metodología más adecuada para estudiar la dinámica del cambio, medir efectos que no se podrían observar con datos puramente de corte transversal o de series de tiempo, y estudiar modelos de comportamiento más complejos.

El panel de datos resultante es corto y balanceado pues el número de empresas supera por mucho el número de años (panel corto), y cada una de las compañías tiene el mismo número de observaciones (panel balanceado) (Gujarati \& Porter, 2009).

Como base se tuvieron los trabajos de autores como Castillo y Moreno (2008), Allayannis y Weston (1998), Jin y Jorion (2006), Guay y Kothari (2002), y Giraldo \& Otros (2017). Se busco entonces, fundamentado en ellos, construir un modelo capaz de conocer la relación y efecto que existe entre los derivados financieros y el valor empresarial, no solo desde su componente decisional (usa o no usa derivados), sino también desde el monto de la estrategia de dichos contratos.

Con esta finalidad, se plantearon 3 modelos de regresión que solo cambiaron en su variable dependiente, en un estilo parecido al planteado por Ahmed, Azevedo y Guney (2013), auto- 
res que usan más de una variable dependiente para sus modelos econométricos. Se propone entonces, medir el valor de las empresas a través de: la Q de Tobin, variable predominante en este tipo de estudios y que mide el valor de las compañías a partir de información de mercado; el índice RONA, variable interna que supone el beneficio de una empresa por el uso de sus activos netos; y el flujo de caja, variable que mide la capacidad de generación de efectivo de una organización y que es usada para estimar el valor de las empresas en consonancia con los modelos actuales de valoración.

Debido al tipo de selección y la muestra, es decir, selección de unidades individuales de corte transversal no extraídas de forma aleatoria y atendiendo a una muestra que reviste el número total de compañías de interés (todas las compañías latinoamericanas que cotizan en ADR's), y estando los sujetos concentrados en una misma región del mundo con características similares, los autores decidieron usar el modelo de panel por efectos fijos por encima del panel por efectos aleatorios. Tampoco se decidió escoger un panel dinámico, ya que al realizar estimadores por Arellano-Bond se encontró que el instrumento principal, el cual es la variable de interés rezagada, no tenía significancia, y a su vez, se pierde esta misma cualidad en algunas de las variables explicativas. También se enfatiza el hecho de que las estimaciones realizadas se desarrollaron incluyendo errores robustos, lo cual soslaya la posibilidad de heteroscedasticidad. En cuanto a autocorrelación, los paneles con pocos años no deberían tener problemas significantes con respecto a este tema (Wooldridge, 2013), aun así, esta es una característica de los datos en los cuales la correlación entre valores de las mismas variables está basada en objetos de interés relacionados, como pasa en el caso de este artículo (Snedecor \& Cochran, 1989). Por último, para minimizar posibles problemas de endogeneidad se estimaron los modelos utilizando como instrumento las variables dependientes rezagadas un periodo.

Cabe resaltar, que esta investigación puede ser considerada como una prolongación del estudio realizado por Saito y Schiozer (2014), aunque con notables diferencias, entre las que se encuentran:

- Se han anexado a la muestra los países de Colombia y Perú.

- El estudio se realiza para los años más recientes (2013 a 2017).

- El foco de la investigación no son los determinantes de los derivados financieros, sino su efecto y comportamiento en el valor empresarial, medido a través de varias variables (Q de Tobin, índice RONA y Flujos de caja).

- Se tienen en cuenta los derivados sobre todo tipo de subyacente (tasa de interés, divisas, commodities, acciones, índices bursátiles, etc.), y finalidad (cobertura, especulación y arbitraje).

- Se cuenta con información del monto de los derivados financieros a valor razonable, y la posición neta de cada una de las empresas en estos instrumentos, lo que evita hacer 
inferencias mediante los montos nocionales o las declaraciones de cada uno de los agentes de la muestra.

Ahora bien, con relación a las variables utilizadas en el modelo, y tomando como base en especial las investigaciones ya referenciadas en este aparte, se evaluaron las siguientes variables independientes y dependientes.

Variables independientes:

- Tamaño de la empresa (X1): esta variable es medida como el logaritmo natural del total de los activos.

- Rentabilidad (X2): esta variable es medida con el ROA, ratio definido como la Utilidad Neta divida por el total de activos.

- Crecimiento de la inversión vía activos fijos (X3): esta variable es medida como la propiedad, planta y equipo (neta) divida el ingreso neto.

- Leverage (X4): se define esta medida como el total de los pasivos divido por el patrimonio.

- Impuestos (X5): este ítem se medirá como el gasto por impuesto dividido el ingreso neto.

- Margen EBITDA (X6): esta variable es medida como el EBITDA divido por los ingresos netos.

- Liquidez (X7): esta variable es medida como el KTO (Capital de trabajo operativo) dividido los pasivos corrientes.

- Productividad del KTNO (X8): esta variable es cuantificada como el KTNO (Capital de trabajo neto operativo) dividido por el ingreso neto.

- Nivel de endeudamiento (X9): esta variable es medida como el pasivo total dividido el activo total.

- Endeudamiento financiero (X10): esta variable es cuantificada como el endeudamiento financiero dividido el ingreso neto.

- Z score de Altman (X11): como medida de estrés financiero, se tiene en cuenta el Z score de Altman .

- Efecto del derivado en balance $(\Delta)$ : está calculada como el valor de la posición neta en derivados a valor razonable (es decir, posición activa o pasiva neta en balance) dividido 
en el total de activos. Esto da una medida del posible efecto en las finanzas que puede tener una empresa en un futuro al cerrarse dichos contratos, y por ende en su valor.

- Efecto del derivado en resultados $(\Phi)$ : el resultado de los derivados financieros en el año puede o bien ser positivo o negativo, dependiendo de qué tan bien se estructuró dicha estrategia, y si se cumplieron las previsiones. Esto impacta directamente en el estado de resultados de la empresa en el aparte de ganancias o pérdidas financieras. Teniendo presente esto, dicha variable es medida como la ganancia o pérdida en resultados en el año dividido el ingreso neto.

- Dummy derivados $(\Omega)$ : variable dummy, 1 si la empresa uso derivados y 0 si no.

Se aclara que de las 300 observaciones realizadas (5 por cada empresa), en 263 de ellas se observa el uso de derivados financieros, mientras que en 37 , no.

Variables dependientes:

- Q de Tobin (Q): es cuantificada como un ratio financiero que mide la relación entre el valor de mercado de una organización y su valor contable. Para Chung y Pruitt (1994), esta variable es medida como la suma entre el valor de mercado del patrimonio (o capitalización bursátil) y el valor en libro del pasivo total, todo esto dividido por el valor en libros del total de activos. Para el modelo 1, se medirá esta variable dependiente como el logaritmo natural de la Q de Tobin.

- RONA (RONA): mide que tan bien una empresa está usando sus activos de operación para generar beneficios. El RONA es medido como la UODI (Utilidad operativa después de impuestos) dividida entre el ANDEO (activos netos de operación). Para el modelo 2, se medirá esta variable como el logaritmo natural del RONA.

- Flujo de caja (FC): basados en ideas propuestas por Deshmukh y Stephen (2005), se plantea la medida del flujo de caja como el logaritmo natural de la razón entre el flujo de caja operativo (EAO) divido por el ANDEO. Lo anterior, suponiendo que el óptimo uso de los activos netos de operación ayuda a la generación de flujos de caja positivo, y por ende, a una apreciación de la empresa. 
Tabla 1

Estadística descriptiva

\begin{tabular}{llcccc}
\hline Variable & & Media & Desv. Est. & Min & Max \\
\hline X1 & Tamaño de la Empresa & 15.74 & 1.44 & 11.94 & 19.60 \\
X2 & Rentabilidad & 0.04 & 0.07 & -0.49 & 0.21 \\
X3 & Crecimiento de la Inversión & 0.77 & 0.61 & 0.01 & 3.99 \\
X4 & Leverage & 2.03 & 5.05 & -30.96 & 51.31 \\
X5 & Impuestos & 0.04 & 0.06 & -0.25 & 0.72 \\
X6 & Mg EBITDA & 0.24 & 0.17 & -0.48 & 1.05 \\
X7 & Liquidez & 1.30 & 1.05 & 0.17 & 8.75 \\
X8 & Productividad del KTNO & 0.25 & 0.25 & -0.33 & 1.85 \\
X9 & Nivel de Endeudamiento & 0.57 & 0.30 & 0.12 & 4.53 \\
X10 & Endeudamiento Financiero & 0.57 & 0.45 & - & 3.08 \\
X11 & Z-Score de Altman & 2.79 & 1.95 & -0.04 & 11.45 \\
$\Delta$ & Efecto Derivado en balance & 0.00 & 0.02 & -0.09 & 0.08 \\
$\Phi$ & Efecto Derivado en resultados & - & 0.02 & -0.19 & 0.21 \\
QT & Q de Tobin & 0.25 & 0.42 & -0.95 & 1.63 \\
RONA & RONA & 0.99 & 0.94 & -0.46 & 1.19 \\
FC & Flujo de Caja & 0.61 & 0.85 & -0.64 & 0.70 \\
\hline
\end{tabular}

Fuente: elaboración propia.

Una vez dispuesta dicha información, el modelo planteado se realizó como una estimación multivariada usando para ello la metodología de panel de datos. Esta estimación como se ha mencionado, consiste en tres regresiones iguales en sus variables independientes, pero distintas en cuanto a sus variables dependientes. De esta manera, las tres regresiones propuestas son como se establecen a continuación:

$$
\begin{aligned}
& \log (\mathrm{Q}) \text { it }=\beta 1+\beta 2 \text { Xit }+\beta 3 \Delta \text { it }+\beta 4 \Phi \text { it }+\beta 5 \Omega \text { it }+\alpha i+\mu \text { it } \\
& \log (\text { RONA }) \text { it }=\beta 1+\beta 2 \text { Xit }+\beta 3 \Delta \text { it }+\beta 4 \Phi \text { it }+\beta 5 \Omega i t+\alpha i+\mu i t \\
& \log (\text { FC)it }=\beta 1+\beta 2 \text { Xit }+\beta 3 \Delta \text { it }+\beta 4 \Phi \text { it }+\beta 5 \Omega \text { it }+\alpha i+\mu i t
\end{aligned}
$$


Donde Q corresponde a la Q de Tobin, RONA al rendimiento sobre los activos netos de operación, FC al flujo de caja, $\mathrm{X}$ a las variables de control, $\Delta$ al efecto en balance de los derivados financieros, $\Phi$ al efecto en resultados de los derivados financieros para ese año, $\Omega$ a la variable Dummy de derivados ( 1 si la empresa usa derivados y 0 en caso contrario), $\alpha$ al efecto no observado o de heterogeneidad y $\mu$ al término error.

El primero de estos modelos procuró medir el efecto de los derivados financieros sobre el valor de la empresa a través de una medida de mercado; por su parte, el segundo modelo hizo lo mismo, pero vía rendimiento o beneficio interno; y por último, se planteó el mismo esquema solo que medido a través del flujo de caja y la capacidad de la empresa para generar este.

\section{Resultados}

En la tabla 2 se encuentran los resultados de las regresiones multivariadas descritas en el aparte anterior (Q de Tobi, RONA y FC). En la tercera, quinta y séptima columna de datos (Coeficientes), se presenta la estimación de los Betas de las variables independientes bajo panel por efectos fijos. Por su parte, la cuarta, sexta y octava columna de datos, se relacionan con la significancia de las variables mediante su P valor a 0.10.

Ahora, y al revisar los resultados, emergen varios hechos de importancia, entre los cuales destaca, a sorpresa de los autores, que para la muestra estudiada, el uso de derivados financieros además de no tener un impacto significante en el valor de las empresas, tiende a ser indirectamente proporcional a este último, pues se encuentra que dicha relación entre variables es negativa. Lo anterior, lleva a resultados parecidos a los encontrados en el trabajo de Jin y Jorion (2006), solo que por cuestiones sustancialmente diferentes. Mientras que estos últimos expresan que la prima por cobertura tiende a desaparecer en un mercado donde los riesgos son fácilmente protegidos por los inversionistas gracias a la diversificación, y que en últimas hacen de las estrategias de cobertura algo inútiles; los autores de este artículo respaldan la idea de que estos resultados se presentan atendiendo a lo inmaduro del mercado latinoamericano en este tipo de temas, al miedo o desconocimiento que aún puedan tener ciertos agentes a la hora de establecer sus portafolios, a la escueta infraestructura para llevar a cabo estas operaciones, a lo débil de los gobiernos corporativos en esta región del mundo, o simplemente a lo reducido de las transacciones a cubrir, pues no habrá que negar el hecho de que la mayoría de compañías latinoamericanas apenas tienen operaciones en el exterior, o las mismas son mínimas a comparación de las que poseen dentro de su campo de influencia.

No se quiere decir con esto que todas las compañías de Latinoamérica puedan ser evaluadas bajo este mismo estándar, sino que para el común, aun falta un gran camino que recorrer para alcanzar la magnitud de organizaciones de otras latitudes en cuanto a operaciones y recursos. 
De manera sobria, se vislumbra un mercado que dista de tener un comportamiento perfecto, con deficiencias difíciles de soslayar en el corto plazo y que frenan de manera estrepitosa el avance de las empresas.

Tabla 2

Resumen de resultados Modelo de panel de datos

\begin{tabular}{|c|c|c|c|c|c|c|c|}
\hline \multicolumn{2}{|l|}{$\mathrm{R}^{\wedge} 2$} & \multicolumn{2}{|c|}{ Modelo 1: Q - Tobin } & \multicolumn{2}{|c|}{ Modelo 2: RONA } & \multicolumn{2}{|c|}{ Modelo 3: FC } \\
\hline \multicolumn{2}{|c|}{ Within } & 0.5914 & & 0.1662 & & 0.1142 & \\
\hline \multicolumn{2}{|c|}{ Between } & 0.5152 & & 0.0605 & & 0.0124 & \\
\hline \multicolumn{2}{|c|}{ Overall } & 0.5301 & & 0.0578 & & 0.0176 & \\
\hline \multicolumn{2}{|c|}{ Variable } & Coeficiente & $\mathrm{P}>\mathrm{ItI}$ & Coeficiente & $\mathrm{P}>\mathrm{ItI}$ & Coeficiente & $\mathrm{P}>\mathrm{ItI}$ \\
\hline $\mathrm{X} 1$ & $\begin{array}{l}\text { Tamaño de la Em- } \\
\text { presa }\end{array}$ & -0.0360942 & 0.478 & -0.9410156 & 0.002 & -0.5996643 & 0.022 \\
\hline $\mathrm{X} 2$ & Rentabilidad & -0.0926276 & 0.673 & -0.1875737 & 0.162 & 0.1698947 & 0.144 \\
\hline $\mathrm{X} 3$ & $\begin{array}{l}\text { Crecimiento de la } \\
\text { Inversión }\end{array}$ & -0.0505746 & 0.354 & -0.5285786 & 0.097 & -0.3868497 & 0.168 \\
\hline $\mathrm{X} 4$ & Leverage & 0.0015946 & 0.482 & 0.0145533 & 0.283 & -0.0003505 & 0.976 \\
\hline $\mathrm{X} 5$ & Impuestos & 0.1652514 & 0.359 & 0.2698358 & 0.799 & 0.1172933 & 0.901 \\
\hline $\mathrm{X} 6$ & Mg EBITDA & 0.1071751 & 0.274 & 0.2249499 & 0.698 & -0.4836993 & 0.344 \\
\hline $\mathrm{X} 7$ & Liquidez & -0.0055923 & 0.796 & -0.0138424 & 0.914 & 0.0011673 & 0.992 \\
\hline $\mathrm{X} 8$ & $\begin{array}{l}\text { Productividad del } \\
\text { KTNO }\end{array}$ & 0.1170644 & 0.333 & -0.1219408 & 0.091 & -0.2446135 & 0.699 \\
\hline X9 & $\begin{array}{l}\text { Nivel de Endeuda- } \\
\text { miento }\end{array}$ & 0.4344671 & 0.000 & 0.0439190 & 0.839 & 0.0195234 & 0.919 \\
\hline $\mathrm{X} 10$ & $\begin{array}{l}\text { Endeudamiento } \\
\text { Financiero }\end{array}$ & 0.0574176 & 0.376 & 0.7273496 & 0.067 & 0.5356497 & 0.116 \\
\hline $\mathrm{X} 11$ & Z-Score de Altman & 0.1280245 & 0.000 & 0.1813201 & 0.079 & 0.0789249 & 0.386 \\
\hline$\Delta$ & $\begin{array}{l}\text { Efecto Derivado en } \\
\text { balance }\end{array}$ & 0.0332190 & 0.968 & 0.2051233 & 0.671 & 0.6371804 & 0.140 \\
\hline$\Phi$ & $\begin{array}{l}\text { Efecto Derivado en } \\
\text { resultado }\end{array}$ & -0.6100201 & 0.071 & -0.2515038 & 0.206 & 0.1898205 & 0.288 \\
\hline$\Omega$ & Dummy Derivado & -0.0239582 & 0.669 & -0.0852330 & 0.797 & -0.1088755 & 0.710 \\
\hline \multirow[t]{2}{*}{$\mathrm{B}$} & Constante & 0.1558583 & 0.842 & 0.1206815 & 0.009 & 0.7399352 & 0.066 \\
\hline & $\begin{array}{l}\text { Variable dependien- } \\
\text { te }(-1)\end{array}$ & 0.1643842 & 0.000 & -0.2970590 & 0.000 & -0.2345419 & 0.005 \\
\hline
\end{tabular}

Fuente: elaboración propia. 
En cuanto a las otras dos variables sobre derivados, se encuentra que el efecto de la estrategia de derivados tanto en balance como en resultados tiende a no ser significativas en ningún modelo, solo pudo probarse esta cualidad en el modelo Q de Tobin, al ser el efecto del derivado en resultados significante. Entre tanto, también se observa que el efecto del derivado en balance tiende a ser positivo en los tres modelos, mientras que los resultados de las estrategias usadas dentro de los años en cuestión fueron de carácter negativo, exceptuado el modelo Flujo de Caja, lo que remarcaría la idea de que las estrategias en derivados financieros producen un incremento en el flujo de caja de las empresas al reducir la volatilidad de estos. En general, lo anterior deja prever que, aunque los resultados de las estrategias en derivados realizadas por las organizaciones estudiadas no fueron eficientes en los años en cuestión, se espera que en un futuro al cerrar los contratos derivados se tienda a cambiar de rumbo, y que los mismos produzcan ganancias, pues existe la posibilidad de que los mencionados tengan un tiempo de vida más allá del corto plazo.

Puede que los resultados hasta aquí descritos parezcan un tanto contradictorios al presentar tan dispares deducciones, pero como lo establecen Castillo y Moreno (2008), el hecho de usar derivados financieros no siempre tiene que ir ligado a un monto importante en estas operaciones, y que no es lo mismo el grado en que se usan a la mera decisión de si utilizarlos o no, es decir, no es lo mismo tomar una posición de un dólar que tomar una posición en un millón de dólares. Por lo tanto, se depara como base de este artículo la idea de que el hecho de solo tomar parte o no en un contrato derivado automáticamente no generará un impacto significativo en el valor, y que es en razón a la cantidad suscrita, a la manera en cómo son estructurados y administrados, lo que demuestra en últimas la utilidad de los mismos.

Con respecto a las variables de control, se contempla que el Z score de Altman y el nivel de endeudamiento son significativos para el modelo Q de Tobin de manera positiva; mientras que para el modelo RONA, el tamaño de la empresa, el crecimiento de la inversión, la productividad del KTNO, el endeudamiento financiero y el mismo Z score de Altman son variables significativas, teniendo las tres primeras una relación positiva y las demás negativa. En última instancia, solo se presenta significancia para el modelo Flujo de Caja en la variable tamaño de la empresa, que a su vez reviste una relación negativa.

No es de sorprender que el Z score de Altman esté relacionado de forma positiva con el valor de una empresa, pues entre más alto sea este indicador se pone de manifiesto la poca factibilidad de que la organización tienda a la quiebra o insolvencia, por lo cual, una compañía se mostrará como más valiosa entre más alejada este de una catástrofe económica. Del mismo modo, un buen grado de endeudamiento financiero, estable y sano, repercutirá positivamente en como es percibida una empresa, no solo por la solidez financiera visible sino también por sus méritos al bajar su costo promedio ponderado de capital al contar con recursos más baratos con terceros que los que ofrecen los mismos accionistas, esto sin contar que puede darse la 
situación en que el mayor nivel de deuda esté asociado a financiar proyectos de valor presente neto positivo que en últimas instancias repercutirán favorablemente.

Con relación al tamaño de la empresa y al crecimiento de la inversión, y su comportamiento enteramente negativo en su mayoría, se puede establecer que empresas el crecimiento no siempre debería atenderse como un signo de mejoramiento o robustez financiera, pues un gran tamaño o un crecimiento irregular y desmedido pueden propender a altos niveles de burocracia corporativa que impidan la flexibilidad y eficiencia en las decisiones, sin contar los altos costos a los cuales deben enfrentarse al mantener una estructura como tal (Allayannis, Lel, $\&$ Miller, 2012). Es entonces importante recalcar que existen crecimientos insostenibles, que a la larga suponen sacrificios importantes y van en contravía del principio básico financiero.

Adicional, la variable productividad del capital de trabajo neto operativo KTNO posee un efecto negativo en el valor de las empresas según los modelos RONA y Flujo de Caja. Esto puede deberse a que su uso o gestión por parte de las organizaciones estudiadas no ha sido el más indicado, lo cual ha producido ineficiencias, y por ende, este resultado dentro de los modelos.

Aunque no significativos para los tres modelos presentados, las variables: impuestos, leverage, $\mathrm{Mg}$ EBITDA, rentabilidad y liquidez han presentado una consistencia media, siendo la primera de carácter positivo en los tres modelos; las siguientes dos, positivas para los modelos Q de Tobin y Rona, y negativa para el modelo Flujo de Caja; y las últimas dos negativas para los modelos dos primeros modelos, y positivas para el tercer modelo.

De estos resultados destacamos que entre menos sea la carga impositiva a la que se enfrenta una compañía, el escudo fiscal sea apropiado, esté apalancada y su margen EBITDA sea capaz de cubrir y atender a todas las obligaciones, una organización se distinguirá como más valiosa, y los individuos del mercado la premiaran con una prima en su importe o tasación. En tanto la variable rentabilidad y liquidez se inclinan a ser negativas en los modelos descritos, lo cual escapa a la comprensión teórica, pues bajo el marco financiero toda compañía con rentabilidad positiva o un buen grado de liquidez debería ser más valiosa que una que no. Esto último, toda vez que la rentabilidad o liquidez generada sea desperdiciada y no se esté usando en proyectos que generen mejores resultados. 
Tabla 3

Resumen de comportamiento y significancia por modelo

\begin{tabular}{|c|c|c|c|c|c|c|c|}
\hline \multicolumn{2}{|c|}{ Variable } & \multicolumn{2}{|c|}{ Modelo 1: Q - Tobin } & \multicolumn{2}{|c|}{ Modelo 2: RONA } & \multicolumn{2}{|c|}{ Modelo 3: FC } \\
\hline & & Significancia & Signo & Significancia & Signo & Significancia & Signo \\
\hline $\mathrm{X} 1$ & Tamaño de la Empresa & - & NS & - & $\mathrm{S}$ & - & $\mathrm{S}$ \\
\hline $\mathrm{X} 2$ & Rentabilidad & - & NS & - & NS & + & NS \\
\hline X3 & $\begin{array}{l}\text { Crecimiento de la } \\
\text { Inversión }\end{array}$ & - & NS & - & $\mathrm{S}$ & - & NS \\
\hline $\mathrm{X} 4$ & Leverage & + & NS & + & NS & - & NS \\
\hline $\mathrm{X} 5$ & Impuestos & + & NS & + & NS & + & NS \\
\hline X6 & Mg EBITDA & + & NS & + & NS & - & NS \\
\hline $\mathrm{X} 7$ & Liquidez & - & NS & - & NS & + & NS \\
\hline $\mathrm{X} 8$ & $\begin{array}{l}\text { Prodctividad del } \\
\text { KTNO }\end{array}$ & + & NS & - & S & - & NS \\
\hline X9 & $\begin{array}{l}\text { Nivel de Endeuda- } \\
\text { miento }\end{array}$ & + & $\mathrm{S}$ & + & NS & + & NS \\
\hline $\mathrm{X} 10$ & $\begin{array}{l}\text { Endeudamiento Fi- } \\
\text { nanciero }\end{array}$ & + & NS & + & S & + & NS \\
\hline $\mathrm{X} 11$ & Z-Score de Altman & + & $\mathrm{S}$ & + & $\mathrm{S}$ & + & NS \\
\hline$\Delta$ & $\begin{array}{l}\text { Efecto Derivado en } \\
\text { balance }\end{array}$ & + & NS & + & NS & + & NS \\
\hline$\Phi$ & $\begin{array}{l}\text { Efecto Derivado en } \\
\text { resultados }\end{array}$ & - & $\mathrm{S}$ & - & NS & + & NS \\
\hline$\Omega$ & Dummy Derivado & - & NS & - & NS & - & NS \\
\hline
\end{tabular}

Fuente: elaboración propia.

\section{Conclusiones}

Este paper estudia el efecto de los derivados financieros en 6 países de Latinoamérica: Argentina, Brasil, Chile, Colombia, México y Perú, usando para ello una muestra de 60 compañías que negocian ADR's en el mercado estadounidense. Los datos de la muestra fueron recolectados de los informes 20-F y 10-K de la Securities and Exchange Commission (SEC), así como de informes trimestrales, semestrales, anuales, y presentaciones y audios de conferencias internacionales, compartidos por cada una de las empresas objeto de la muestra en sus páginas oficiales para inversionistas en Internet. También se hizo uso del software Economatica para 
recolectar otra información de mercado importante o validar ciertos datos. El estudio entrama los años comprendidos entre el 2013 al 2017.

La metodología de estimación se basó en regresiones usando paneles de datos, específicamente el panel de datos de efectos fijos. Como variables dependientes que midieran el valor de las compañías, se tuvieron en cuenta la Q de Tobin (medida del valor a través del mercado), el índice RONA (medida del valor a través del rendimiento) y el flujo de caja (medida del valor a través de la capacidad de generación de efectivo). Por su parte, las variables independientes o explicativas fueron las siguientes: tamaño de la empresa, rentabilidad, crecimiento de la inversión vía activos fijos, leverage, impuestos, margen EBITDA, liquidez, productividad del KTNO, nivel de endeudamiento, endeudamiento financiero, Z Score de Altman, efecto del derivado en balance, efecto del derivado en resultados, y una dummy para el uso de derivados.

Los datos arrojados en los 3 modelos tratados (Q de Tobin, RONA y Flujo de Caja) establecen consistentemente que para la muestra y los años seleccionados, el uso de derivados financieros y el grado manejado en dichos contratos, no repercute significativamente en el valor de las mismas, y que los mismos pueden generar perdidas de valor no significativas aunque en el futuro se espere lo contrario, de allí la dualidad que para los modelos mencionados la relación entre el efecto de los derivados en el resultado sea negativo con respecto al valor, pero que el efecto del derivado en balance sea lo contrario, es decir positivo.

Habrá que aclarar que, aunque las variables dependientes sometidas a estudio son parcialmente diferentes, comparten el hecho de ser medidas de valor usadas en todo el mundo, y que en la praxis se unen para dar una perspectiva más completa del acervo económico de una organización, siendo la Q de Tobin una perspectiva de mercado, y el RONA y el Flujo de Caja, una perspectiva más interna. Esto da pie para enfatizar en que los resultados han mostrado que aun viendo el tema de los derivados desde distintas perspectivas se logra llegar a una conclusión, la cual no es otra más que la ya mencionada carencia de impacto de estos en el valor empresarial. Entre las explicaciones de esto, podemos mencionar la inmadurez e imperfección del mercado latinoamericano con respecto al nicho de los derivados financieros, al desconocimiento por parte de estas empresas de los productos existentes o de estrategias que pueden iniciar con los mismos, del capital humano no preparado en estos temas, de lo costoso de levantar una infraestructura para llevar a cabo estas operaciones o contratar personal idóneo de alto impacto, de la debilidad de los gobiernos corporativos o la poco protección a los inversionistas en esta región del mundo, problemas de información asimétrica, del miedo en cuanto a la complejidad de estas herramientas y a que un mal uso puede llevar a la bancarrota, o por último, al poco interés de usarlos por lo mínimo de las transacciones a cubrir.

Queremos remarcar el hecho de que en Latinoamérica el tema de derivados no es conocido ampliamente o tienen una connotación de tabú, ostentando para los gerentes un estigma de 
complejos, costosos o hasta peligrosos, y por lo mismo, no se ha visto tan aceptado y aprovechado en las latitudes estudiadas.

En todo caso, se rechaza la hipótesis planteada en este trabajo al evidenciarse que para los tres modelos la significancia es mínima y negativa.

Por último, reforzamos la marcada tendencia en la literatura especializada de manifestar la falta de consenso de las investigaciones empíricas, o la insuficiencia de estudios que vayan más allá de los realizados hasta ahora. Y de este modo, destacamos la importancia de seguir con las investigaciones sobre el tema de derivados financieros, pues la trascendencia de estos últimos como estrategia corporativa se acrecienta cada vez más en un mundo con altos niveles de volatilidad. Por lo tanto, se propone para el caso, que se tengan en cuenta variables diferentes a las aquí analizadas, haciendo un conjunto más heterogéneo, donde se presenten variables internas y externas, tales como la volatilidad del mercado, tasas de interés, tasas de cambio, yield de dividendos, KZ index, WACC (costo de la deuda y patrimonio), entre otros. Adicional, se aboga por utilizar muestras más amplias para el desarrollo de este tipo de estudios en esta región del mundo (Latinoamérica), debido que este trabajo y previos, se concentran en un pequeño porcentaje del total de las empresas de los países que hacen parte de los trabajos realizados, lo cual también conlleva el reto de una metodología que mida el valor de las compañías que no están en bolsa, las cuales son mayoría y representan otras características de este tipo de regiones.

\section{Referencias}

Ahmed, H., Azevedo, A., \& Yilmaz, G. (2013). The Effect of Hedging on Firm Value and Perfomance: Evidence from the Nonfinancial UK Firms. Cottingham. In European Financial Management Association Annual Meetings Proceedengs 2014 (Vol. 44, pp. 1-5). Italy, Rome. Disponible en: http://www.efmaefm.org/0EFMAMEETINGS/EFMA ANNUAL MEETINGS/2014 Rome/papers/EFMA2014_0342_fullpaper.pdf - Consultado: $04 / 01 / 2019$

Allayannis, G., \& Weston, J. (1998). The use of foreing currency derivatives and firm market value. Virginia. https://doi.org/10.1093/rfs/14.1.243

Allayannis, G., Lel, U., \& Miller, D. (2012). The use of foreign currency derivatives, corporate governance, and firm value around the world. Journal of international economics vol 87.(1), 65-79. http://doi.org/10.1016/j. jinteco.2011.12.003

Bartram, S., Brown, G., \& Fehle, F. (2009). International evidence on financial derivatives usage. Financial Management vol 38 no 1, 185-206. https://doi.org/10.1111/j.1755-053X.2009.01033.x

Bartram, S., Brown, G., \& Conrad, J. (2011). The effects of derivatives on firm risk and value. The journal of financial and quantitative analysis vol 46 no 4, 967-999. 46(4), 967-999. http://doi.org/10.1017/S0022109011000275

Berkman, H., \& Bradbury, M. (1996). Empirical evidence on the corporate use of derivates. Financial Management Vol 25 No 2, 5-13. The Journal of the Financial Management Association, 25(2), 5. http://doi. org $/ 10.2307 / 3665985$ 
Berrospide, J., Purnanandam, A., \& Rajan, U. (2008). Corporate Hedging, Investment and Value. Washington D.C. http://doi.org/10.2139/ssrn.1009657

Brown, G. W. (2001). Managing foreign exchange risk with derivatives. Journal of Financial Economics, 60(2), 401-448. http://doi.org/10.1016/S0304-405X(01)00049-6

Brown, G., \& Crabb, H. (2002). Are firms successful at selective hedging? Carolina del Norte: University of North Carolina. http://doi.org/10.1086/508004

Castillo, A., \& Moreno, D. (2008). Uso de derivados cambiarios y su impacto en el valor de empresas: el caso de empresas chilenas no financieras. Estudios de administración Vol 15 no. 1, 1-30. Disponible en: http://estudiosdeadministracion.unegocios.cl/index.php/2008-vol-15-no-1.html - Consultado: 07/01/2019

Chung, K., \& Pruitt, S. (1994). A simple approximation of Tobin's Q. Financial Management, 70-74. http://doi. org $/ 10.2307 / 3665623$

Clark, E., Judge, A., \& Wing, N. (2006). The determinants and value effects of corporate hedging: an empirical study of Hong Kong and Chinese firms. London: Accounting and finance group Middlesex University, 14(3), 445-469. http://doi.org/10.1111/j.1468-036X.2007.00360.x

Coronas Dueñas, J. A. (2012). Análisis de la utilización de derivados financieros en las empresas no financieras mexicanas y su efecto en las cotizaciones bursátiles. Revista Atlántica de Economía Vol 1.

Corzo, R., \& Balbi, M. (2007). Los instrumentos financieros derivados: qué son, para qué sirven y cómo funcionan. Themis Revista de Derecho no 54, 205-225. Disponible en: https://dialnet.unirioja.es/servlet/articulo?codigo=5110694 - Consultado: 04/01/2019

Cowan, K., Hansen, E., \& Herrera, L. O. (2006). Currency mismatches in chilean nonfinancial corporations. En B. C. Chile, External vulnerability and preventive policies. http://doi.org/10.2139/ssrn.674081

Cummins, D., Phillips, R., \& Sthepen, S. (2001). Derivatives and corporate risk management: participation and volume decision in the insurance industry. The journal of risk and insurance vol 68 no 1, 51-92. http://doi. org/10.2307/2678132.

Deshmuk, S., \& Stephen, V. (2005). Investments, Cash Flow and Corporate Hedging. The Journal of Corporate Finance, 628-644. Disponible en: https://ssrn.com/abstract=2512875 - Consultado: 25/12/2018.

Dhanani, A., Fifield, S., Helliar, C., \& Stevenson, L. (2007). Why Uk Companies Hedge Interest Rate Risk. Studies in Economics and Finance 24, 72-90. https://doi.org/10.1108/10867370710737391

Fauver, L., \& Naranjo, A. (2010). Derivative usage and firm value: the influence of agency costs and monitoring problems. Journal of corporate finance vol 16, 719-735. https://doi.org/10.1016/j.jcorpfin.2010.09.001

Fierros Villanueva, P. A. (2012). El mercado de derivados financieros y su impacto en el valor de las empresas en México. Tijuana: Colegio de la frontera Norte. Disponible en: https://www.colef.mx/posgrado/wp-content/ uploads/2014/03/TESIS-Fierros- Villanueva-Perla-Aceleth.pdf - Consultado: 15/12/2018

Gay, G. D., \& Nam, J. (1998). The underinvestment problem and corporate derivatives use. Financial Management, 27(4), 53-69. http://doi.org/10.2307/3666413

Géczy, C., Minton, B., \& Schrand, C. (1997). Why Firms Use Currency Derivatives. The Journal of Finance, 52(4), 1323-1354. http://doi.org/10.1111/j.1540-6261.1997.tb01112.x

Giraldo, C. A., Gonzáles, J., Vesga, C., \& Ferreira, D. (2017). Coberturas financieras con derivados y su incidencia en el valor de mercado en empresas colombianas que cotizan en bolsa. Contaduría y Administración Vol 62, 1572-1590. http://doi.org/10.1016/j.cya.2017.04.008

Graham, J., \& Smith, C. (1999). Tax incentives to hedge. Journal of finance vol 54, 2241-2262. https://doi. org/10.1111/0022-1082.00187

Graham, J. R., \& Rogers, D. A. (2002). Do Firms Hedge in Response to Tax Incentives? The Journal of Finance, 57(2), 815-839. http://doi.org/10.1111/1540-6261.00443

Guay, W. (1999). The impact of derivatives on firm risk: an empirical examination of new derivatives user. Journal of accounting and economics vol 26, 319-351. https://doi.org/10.1016/S0165-4101(98)00032-9 
Guay, W., \& Kothari, S. (2002). How much do firms hedge with derivatives? Financial economics, 423-461 . https:// doi.org/10.1016/S0304-405X(03)00179-X

Gujarati, D., \& Porter, D. (2009). Econometría. México DF: McGraw-Hill.

Hentschel, L., \& Kothari, S. (2001). Are corporations reducing or taking risks with derivatives? Journal of financial and quiantitive analysis vol 36, 93-118. https://doi.org/10.2307/

Jin, Y., \& Jorion, P. (2006). Firm Value and Hedging : Evidence from U.S. Oil and Gas Producers. Journal of Finance, 61(2), 893-919. http://doi.org/10.1111/j.1540-6261.2006.00858.x

Kozikowski, Z. (2007). Finanzas internacionales. Ciudad de México: McGraw-Hill Interamerican. Disponible en: https://www.uv.mx/personal/clelanda/files/2016/03/Kozikowski-Z-2013-Finanzas-Internacionales.pdf - Consultado: 24/12/2018

Modigliani, F., \& Miller, M. H. (1958). The cost of capital, corporation finance and theory of investment. The American Economic Review Vol 48 No 3, 261-297. http://doi.org/10.1080/17446540802345448

Murillo, C., Chen, L., \& Hong, Z. (2011). The real and financial implications of corporate hedging. The Journal of Finance Vol 65 no 5, 1615-1647. http://doi.org.10.3386/w16622

Nicos, A. S., Barrese, J., \& Wang, P. (2008). The Impact of Cash Flow Volatility on Systematic Risk. Journal of insurance issues vol 31 no 1, 43-71. Disponible en: https://www.jstor.org/stable/41946281 - Consultado: $17 / 12 / 2018$

Place, J., \& Gray, S. (2003). Derivados financieros. México D.F.: Centro de estudios monetarios latinoamericanos.

Saito, R., \& Schiozer, R. (2009). Why do latin american firms manage currency risk. Emerging markets finance and trade vol 45 no 1, 49-71. http://doi.org/10.2139/ssrn.884210

Sarmas, P. (2005). Elements of corporate hedging policy. Southwest Business and Economics Journal 20042005. Disponible en: http://www.cameron.edu/uploads/TC/z9/TCz9XbqJ5uavQcWtswogZg/Vol-13-Elements-Of-Corporate-Hedging-Policy.pdf - Consultado: 27/12/2018

Schiozer, R. F., \& Saito, R. (2009). The Determinants of Currency Risk Management in Latin American Nonfinancial Firms. Emerging Markets Finance and Trade, 45(1), 49-71. http://doi.org/10.2753/REE1540-496X450104

Schiozer, R. F., \& Saito, R. (2014). The determinants of currency risk management in Latin American nonfinancial firms. Emerging markets finance and trade 45, 49-71. Disponible en: https://www.jstor.org/stable/27750652 Consultado: 18/12/2018

Smith, C., \& Stulz, R. (1985). The determinants of firms hedging policies. Journal of financial and quantitive analysis 20, 391-405. http://doi.org/10.2307/2330757

Snedecor, G., \& Cochran, W. (1989). Statistical Methods. Blackwell. https://doi.org/10.1086/334842

Tim, A., \& Chitru, F. (2006). Hedging, speculation and shareholder value. Journal of Financial Economics 81, 283309. https://doi.org/10.1016/j.jfineco.2005.03.014

Wooldridge, J., (2013). Introducción a la econometría. Quinta edición. Cengage Learning. Universidad de Michigan. 